\title{
The Gelfand map and symmetric products
}

\author{
V.M.Buchstaber and E.G.Rees
}

There are many instances of the principle that if $A$ is an algebra of functions on $X$, then every ring homomorphism $A \rightarrow \mathbf{C}$ is given by evaluation at a particular $x \in X$. Examples are the nullstellensatz in algebraic geometry and the result of Gelfand when $X$ is a compact Hausdorff space. In these cases one can regard $X$ as being included in $\operatorname{Hom}(A, \mathbf{C})$ as the set of those $f: A \rightarrow \mathbf{C}$ which satisfy the set of equations $f(x y)=f(x) f(y)$ indexed by $(x, y) \in X \times X$. In this paper we introduce the corresponding equations for the symmetric products of $X$. We show that, in these examples, $\operatorname{Sym}^{n}(X)$ is included in $\operatorname{Hom}(A, \mathbf{C})$ as the set of those $f$ that satisfy these more complicated equations.

Given a linear map $f: A \rightarrow \mathbf{C}$ we consider certain maps which can be regarded as "higher" versions of $f$ and are denoted $\Phi_{n}(f): A^{\otimes n} \rightarrow \mathbf{C}$, their definition is based on formulae used by G. Frobenius [Frol]. The subset $\Phi_{n}(A) \subset \operatorname{Hom}(A, \mathbf{C})$ of the space of all linear maps $\operatorname{Hom}(A, \mathbf{C})$ consisting of those $f$ for which $\Phi_{n+1}(f)=0$ and $f(1)=n$ is particularly interesting and we will develop its properties; $\Phi_{1}(A)$ is the set of ring homomorphisms. When $A$ is an algebra of functions on a space $X$ the sets $\Phi_{n}(A)$ are closely related to the symmetric product $\operatorname{Sym}^{n}(X):=X^{n} / \Sigma_{n}$. The case $n=1$ is classical, if $A$ is a separating algebra of functions on a compact space $X$ then $\Phi_{1}(A)$ is the set of algebra homomorphisms and, by the Gelfand transform, this is homeomorphic to $X$.

In many cases, we can identify $\Phi_{n}(A)$ with the set of maps that can be written as the sum of $n$ ring homomorphisms. In particular, when $X$ a compact Hausdorff space and $A=C(X)$ is the ring of complex valued continuous functions on $X, \Phi_{n}(A)$ is precisely the set of linear maps that can be written as the sum of $n$ ring homomorphisms and so can be identified with $\operatorname{Sym}^{n}(X)$. The analogous result holds when $A$ is a finitely generated commutative algebra. In particular, when $X=\mathrm{C}^{m}$ and the algebra is the ring $A=\mathbf{C}\left[u_{1}, u_{2}, \ldots, u_{m}\right]$ of polynomial functions on $X$ we prove that $\Phi_{n}(A)$ is the symmetric product $\operatorname{Sym}^{n}\left(\mathbf{C}^{m}\right)$. A by product is that the embedding $\Phi_{n}(A) \subset \operatorname{Hom}(A, \mathbf{C})$ can be described by specific equations, we study them in another paper. 
The equations that define $\Phi_{n}$ can be described in several ways and can be derived from formulae introduced by Frobenius [Fro1 to define the $k$ characters of a finite group. These formulae have also been used more recently (eg [For] ) in the study of relations in matrix algebras. In the context of these works, the case where $A$ is non-commutative was of primary interest; indeed such functions were often trivial in the commutative case. In contrast, in this paper we concentrate our attention on the commutative case.

Our initial interest in these ideas came from [BR1] [BR2] where the diagonal of an $n$-Hopf algebra (the analogue of a Hopf algebra for an $n$-valued group) is characterised as being an $n$-ring homomorphism. Professor John McKay kindly pointed out to us that there were similarities to the formulae introduced by Frobenius, and indeed there is a precise relationship [BR3].

The paper is divided into sections:

1. Proves an identity satisfied by partitions of sets and which may be of independent interest.

2. Introduces Frobenius transformations and develops some of their basic properties.

3. Sets up the relationship with symmetric products and proves the basic theorem for finite sets, affine varieties and compact Hausdorff spaces.

\section{$\S 1$ An identity on partitions}

If $\sigma$ is a permutation of a set $X$, there is a partition of $X$ given by the orbits of the action of the group generated by $\sigma$. Clearly, two permutations giving the same partition have the same cycle type and hence the same sign; given a partition $\pi$, let $\epsilon(\pi)$ denote this sign and let $n(\pi)$ be the number of permutations that give rise to $\pi$, so if the parts of $\pi$ are $P_{1}, P_{2}, \ldots, P_{k}$ then $n(\pi)=\prod_{i=1}^{k}\left(\# P_{i}-1\right)$ !.

Let $\mathcal{P}(X)$ denote the free abelian group on the set of all partitions of $X$ and

$$
\chi(X)=\sum \epsilon(\pi) n(\pi) \pi \in \mathcal{P}(X)
$$

where the sum is over all the partitions of $X$.

If $\pi_{1}, \pi_{2}$ are partitions of $X, Y$ respectively, then one has a natural partition $\pi_{1} \pi_{2}$ of the disjoint union $X \sqcup Y$. So, one can define $\chi(X) \chi(Y) \in$ $\mathcal{P}(X \sqcup Y)$. 
If $g: X \rightarrow Y$ is a map and $\pi$ is a partition of $Y$ one has, by taking inverse images of the parts of $\pi$, a partition $g^{*} \pi$ (they have the same number of parts if $g$ is surjective) and hence a homomorphism $g^{*}: \mathcal{P}(Y) \rightarrow \mathcal{P}(X)$.

\section{(1.1) Definition}

A partial pairing $\phi$ between two sets $X, Y$ is a bijection $\phi: X_{\phi} \rightarrow Y_{\phi}$ between subsets $X_{\phi} \subset X$ and $Y_{\phi} \subset Y$. Given a partial pairing $\phi$, define an equivalence relation on $X \sqcup Y$ by $x \sim y$ if $\phi(x)=y$. We denote the quotient set by $X \sqcup_{\phi} Y$ (its cardinality is $\# X+\# Y-\# X_{\phi}$ ) and the quotient map by $q_{\phi}: X \sqcup Y \rightarrow X \sqcup_{\phi} Y$.

\section{(1.2) Proposition}

If $X, Y$ are disjoint, then

$$
\sum q_{\phi}^{*} \chi\left(X \sqcup_{\phi} Y\right)=\chi(X) \chi(Y)
$$

where the sum is over all partial pairings $\phi$ between $X$ and $Y$, including the 'empty' partial pairing.

$\underline{\text { Proof }}$

Let $\pi=P_{1} \sqcup P_{2} \sqcup \ldots \sqcup P_{k}$ be a partition of $X \sqcup Y$ where $P_{j}=\left\{x_{1 j}, x_{2 j}, \ldots, x_{m_{j}}, y_{1 j}, y_{2 j}, \ldots, y_{n_{j} j}\right\}$ with $x_{i j} \in X, y_{i j} \in Y$ and let

$$
c(m, n, \ell)=(-1)^{m+n-\ell-1}(m+n-\ell-1) ! \ell !\left(\begin{array}{c}
m \\
\ell
\end{array}\right)\left(\begin{array}{l}
n \\
\ell
\end{array}\right) .
$$

Then the coefficient of the partition $\pi$ that appears from terms arising from pairing along subsets of cardinality $\ell$ is

$$
\sum_{\ell_{1}+\ell_{2}+\ldots+\ell_{k}=\ell} c\left(m_{1}, n_{1}, \ell_{1}\right) c\left(m_{2}, n_{2}, \ell_{2}\right) \ldots c\left(m_{k}, n_{k}, \ell_{k}\right) .
$$

Hence, the coefficient of $\pi$ in the left hand side of the expression in Proposition (1.2) is

$$
c_{\pi}=\sum_{\ell_{1}=0}^{\min \left(m_{1}, n_{1}\right)} \sum_{\ell_{2}=0}^{\min \left(m_{2}, n_{2}\right)} \ldots \sum_{\ell_{k}=0}^{\min \left(m_{k}, n_{k}\right)} c\left(m_{1}, n_{1}, \ell_{1}\right) c\left(m_{2}, n_{2}, \ell_{2}\right) \ldots c\left(m_{k}, n_{k}, \ell_{k}\right)
$$

To evaluate this sum we let

$$
d(m, n, \ell)=\frac{\left(\begin{array}{c}
m \\
\ell
\end{array}\right)\left(\begin{array}{l}
n \\
\ell
\end{array}\right)}{\left(\begin{array}{c}
m+n-1 \\
\ell
\end{array}\right)}=(-1)^{m+n-1}(-1)^{\ell} \frac{c(m, n, \ell)}{(m+n-1) !}
$$


and

$$
P_{m, n}(t)=\sum_{\ell=0}^{\min (m, n)} d(m, n, \ell)(-t)^{\ell}
$$

then

$$
c_{\pi}=\epsilon(\pi) \prod_{r=1}^{r=k}\left(m_{r}+n_{r}-1\right) ! P_{m_{r}, n_{r}}(1)
$$

The polynomial $P_{m, n}(t)$ is a hyper-geometric polynomial, being a solution of the differential equation

$$
t(1-t) y^{\prime \prime}(t)-(m+n-1)(1-t) y^{\prime}(t)-m n y(t)=0 .
$$

Therefore, by substituting $t=1$ into the differential equation one has $P_{m, n}(1)=$ 0 for $\min (m, n)>0$.

Hence, $c_{\pi}=0$ unless each part of $\pi$ consists either entirely of $x$ 's or entirely of $y$ 's. In this case, let the partition be $\pi_{1} \pi_{2}$; its coefficient in the term $\chi(X) \chi(Y)$ is $\epsilon\left(\pi_{1}\right) n\left(\pi_{1}\right) \epsilon\left(\pi_{2}\right) n\left(\pi_{2}\right)$ (it can only occur once in the product). In the left hand side of the equation in Proposition (1.2) the only term in which $\pi_{1} \pi_{2}$ appears is $\chi(X \sqcup Y)$ and its coefficient is $\epsilon\left(\pi_{1} \pi_{2}\right) n\left(\pi_{1} \pi_{2}\right)=$ $\epsilon\left(\pi_{1}\right) n\left(\pi_{1}\right) \epsilon\left(\pi_{2}\right) n\left(\pi_{2}\right)$.

The result of Proposition (1.2) now follows.

\section{$\S 2$ Frobenius transformations}

In [Fro1] and [Fro2], G. Frobenius introduced the $k$-characters of a finite group and they have been studied again more recently (e.g. Joh, HJ). In [BR3] we extended the concept to a broader context and called them Frobenius transformations. Here we consider the case where the algebras on which they are defined and in which they take values are both commutative. These transformations have interesting applications in these case despite the fact that the $k$-characters considered by Frobenius vanish for irreducible representations of finite abelian groups.

Following [For], where the Frobenius formula for the $k$-character is reformulated, write a permutation $\sigma \in \Sigma_{n+1}$ as a product of disjoint cycles (including those of length one)

$$
\sigma=\gamma_{1} \gamma_{2} \ldots \gamma_{q}
$$

If $f: A \rightarrow B$ is linear and $\gamma$ is the cycle $\left(r_{1} r_{2} \ldots r_{k}\right)$ write $f_{\gamma}\left(a_{1}, a_{2}, \ldots a_{n+1}\right)=$ $f\left(a_{r_{1}} a_{r_{2}} \ldots a_{r_{k}}\right)$. (Note that this also works for non-commutative algebras $A$ provided $f$ is 'trace-like' because in that case the value of $f_{\gamma}\left(a_{1}, a_{2}, \ldots a_{n+1}\right)$ 
depends only on the cycle $\gamma$ and is independent of the way that it is written in terms of the $x$ 's.) Now write

$$
f_{\sigma}=f_{\gamma_{1}} f_{\gamma_{2}} \ldots f_{\gamma_{q}}
$$

(2.1) Definition For a linear map $f: A \rightarrow B$ where $A, B$ are commutative algebras, the map $\Phi_{m}(f): A^{\otimes m} \rightarrow B$ is defined by

$$
\Phi_{m}(f)\left(a_{1}, a_{2}, \ldots, a_{m}\right)=\sum_{\sigma \in \Sigma_{m}} \epsilon(\sigma) f_{\sigma}\left(a_{1}, a_{2}, \ldots, a_{m}\right) .
$$

Remark The map $\Phi_{m}(f)$ is clearly symmetric and multilinear.

We can define $f(\chi(X))$ as follows :

(2.2) Lemma If $X=\left(a_{1}, a_{2}, \ldots, a_{m}\right)$ and $\chi(X)$ is as defined in $\S 1$ then $\Phi_{m}(f)\left(a_{1} \otimes a_{2} \otimes \ldots a_{m}\right)=f(\chi(X))$.

$\underline{\text { Proof }}$

Rewriting the above definition, if $P=\left[a_{i_{1}}, a_{i_{2}}, \ldots, a_{i_{r}}\right]$ is a multi-subset of the multi-set $X=\left[a_{1}, a_{2}, \ldots, a_{m}\right] \subset A$ then $f_{P}\left(a_{1}, a_{2}, \ldots, a_{m}\right)=f\left(a_{i_{1}} a_{i_{2}} \ldots a_{i_{r}}\right)$ and if $\pi=P_{1} \sqcup P_{2} \sqcup \ldots \sqcup P_{k}$ is a partition of $X$, we have $f_{\pi}=f_{P_{1}} f_{P_{2}} \ldots f_{P_{k}}$ and $\Phi_{m}(f)=\sum \epsilon(\pi) n(\pi) f_{\pi}$.

We now recall the inductive definition based on that used by Frobenius.

(2.3) Definition Define, inductively, for $n \in \mathbf{N}$, linear maps $\Phi_{n}(f)$ : $A^{\otimes n} \rightarrow B$ starting with $\Phi_{1}(f)=f, \quad \Phi_{2}(f)\left(a_{1}, a_{2}\right)=f\left(a_{1}\right) f\left(a_{2}\right)-f\left(a_{1} a_{2}\right)$ and for $n \geq 2$ as follows :

$\Phi_{n+1}(f)\left(a_{1}, a_{2}, \ldots, a_{n+1}\right)=f\left(a_{1}\right) \Phi_{n}(f)\left(a_{2}, a_{3}, \ldots, a_{n+1}\right)-$

$\Phi_{n}(f)\left(a_{1} a_{2}, \ldots, a_{n+1}\right)-\Phi_{n}(f)\left(a_{2}, a_{1} a_{3}, \ldots, a_{n+1}\right)-\ldots-\Phi_{n}(f)\left(a_{2}, a_{3}, \ldots, a_{1} a_{n+1}\right)$.

It is a simple consequence of Proposition (1.2) with $X=\left\{a_{1}\right\}, Y=\left\{a_{2}, a_{3}, \ldots, a_{n+1}\right\}$ that the two definitions (2.1) and (2.3) are the same.

Remark It follows immediately from the inductive definition that if $f$ satisfies $\Phi_{n}(f) \equiv 0$ then $\Phi_{n+1}(f) \equiv 0$.

(2.4) Lemma If $B$ is a domain and $\Phi_{n+1}(f) \equiv 0$ but $\Phi_{n}(f) \not \equiv 0$ then $f(1)=n$.

$\underline{\text { Proof }}$ 
Let $a_{1}=1$ then, using the inductive definition, we get

$$
0=\Phi_{n+1}(f)\left(1, a_{2}, a_{3}, \ldots, a_{n+1}\right)=[f(1)-n] \Phi_{n}(f)\left(a_{2}, a_{3}, \ldots, a_{n+1}\right) .
$$

But, since $\Phi_{n}(f) \not \equiv 0$, there are $a_{2}, a_{3}, \ldots, a_{n+1} \in A$ such that $\Phi_{n}(f)\left(a_{2}, a_{3}, \ldots, a_{n+1}\right) \neq 0$.

(2.5) Corollary If $f: A \rightarrow B$ satisfies $\Phi_{n+1}(f) \equiv 0$ and $B$ is a domain then $f(1) \in\{0,1,2, \ldots, n\}$.

Proof

This follows by a simple induction from Lemma (2.4).

(2.6) Definition $A$ linear map $f: A \rightarrow B$ is a Frobenius $n$-homomorphism if $\Phi_{n+1}(f) \equiv 0$ and $f(1)=n$.

Remark When $B$ is a domain, every Frobenius 1-homomorphism $f: A \rightarrow B$ is a ring homomorphism.

(2.7) Proposition If $B$ is a domain then a linear map $f: A \rightarrow B$ such that $\Phi_{n+1}(f) \equiv 0$ and $f(1)=k \leq n$ is a Frobenius $k$-homomorphism .

Proof

Applying the inductive formula to $\Phi_{n+1}(f)\left(1, a_{2}, \ldots, a_{n+1}\right)=0$ gives,

$$
(k-n) \Phi_{n}(f)\left(a_{2}, \ldots, a_{n+1}\right)=0
$$

so $f$ is a Frobenius $k$-homomorphism. The result follows by induction.

We denote the sub-algebra of symmetric tensors in $A^{\otimes n}$ by $\mathcal{S}^{n} A$. The map $\Phi_{n}(f) / n$ ! restricted to $\mathcal{S}^{n} A$ has a multiplicative property:

(2.8) Theorem If $f: A \rightarrow B$ is a Frobenius n-homomorphism, then the map defined by

$$
\frac{\Phi_{n}(f)}{n !}: \mathcal{S}^{n} A \rightarrow B
$$

is a ring homomorphism.

$\underline{\text { Proof }}$

A typical element of $\mathcal{S}^{n} A$ is

$$
\mathbf{a}=\sum_{\sigma \in \Sigma_{n}} a_{\sigma(1)} \otimes a_{\sigma(2)} \otimes \ldots \otimes a_{\sigma(n)}
$$

and so the product of two such elements is

$$
\mathbf{a b}=\sum_{\sigma_{1}, \sigma_{2} \in \Sigma_{n}} a_{\sigma_{1}(1)} b_{\sigma_{2}(1)} \otimes a_{\sigma_{1}(2)} b_{\sigma_{2}(1)} \otimes \ldots \otimes a_{\sigma_{1}(n)} b_{\sigma_{2}(n)}
$$


By Lemma (2.2), if $X=\left(a_{1}, a_{2}, \ldots, a_{n}\right), Y=\left(b_{1}, b_{2}, \ldots, b_{n}\right)$ we have

$$
\Phi_{n}(f)\left(a_{1}, a_{2}, \ldots, a_{n}\right) \Phi_{n}(f)\left(b_{1}, b_{2}, \ldots, b_{n}\right)=f(\chi(X)) f(\chi(Y)) .
$$

Regarding $X, Y$ as disjoint we have

$$
f(\chi(X)) f(\chi(Y))=f(\chi(X) \chi(Y))
$$

By Proposition (1.2) one has

$$
f(\chi(X) \chi(Y))=\sum_{\phi \in \Sigma_{n}} f\left(q_{\phi}^{*} \chi\left(X \sqcup_{\phi} Y\right)\right.
$$

since $f$ is a Frobenius $n$-homomorphism the only terms on the right hand side which are non-zero are those where $\phi: X \rightarrow Y$ is a permutation. By Lemma (2.2),

$$
\sum_{\phi \in \Sigma_{n}} f\left(q_{\phi}^{*} \chi\left(X \sqcup_{\phi} Y\right)\right)=\sum_{\phi \in \Sigma_{n}} \Phi_{n}(f)\left(a_{1} b_{\phi(1)}, a_{2} b_{\phi(2)}, \ldots, a_{n} b_{\phi(n)}\right) .
$$

Hence adding all the relevant terms we get

$$
\begin{aligned}
& \Phi_{n}(f)(\mathbf{a}) \Phi_{n}(f)(\mathbf{b}) \\
= & \sum_{\sigma_{1}, \sigma_{2}, \sigma_{3} \in \Sigma_{n}} \Phi_{n}(f)\left(a_{\sigma_{1}(1)} b_{\sigma_{2}(1) \sigma_{3}(1)}, a_{\sigma_{1}(2)} b_{\sigma_{2}(2) \sigma_{3}(2)}, \ldots, a_{\sigma_{1}(n)} b_{\sigma_{2}(n) \sigma_{3}(n)}\right) \\
= & n ! \sum_{\sigma_{1}, \sigma_{2} \in \Sigma_{n}} \Phi_{n}(f)\left(a_{\sigma_{1}(1)} b_{\sigma_{2}(1)}, a_{\sigma_{1}(2)} b_{\sigma_{2}(2)}, \ldots, a_{\sigma_{1}(n)} b_{\sigma_{2}(n)}\right)=n ! \Phi_{n}(f)(\mathbf{a b})
\end{aligned}
$$

It is easy to check that $\Phi_{n}(f)(1,1, \ldots, 1)=n$ !.

(2.9) Theorem If $f, g$ are Frobenius $m$ - and n-homomorphisms respectively, then $f+g$ is a Frobenius $(m+n)$-homomorphism.

(2.10) Corollary If $f: A \rightarrow B$ is the sum of $n$ ring homomorphisms $f_{i}: A \rightarrow B, 1 \leq i \leq n$ then $f$ is a Frobenius $n$-homomorphism.

To prove (2.9) it is convenient to use polarisation ([W], Chapter II) to study the properties of $\Phi_{n}(f)$; this means that because $\Phi_{n}(f)\left(a_{1}, a_{2}, \ldots, a_{n}\right)$ is both multi-linear and symmetric it is enough to calculate using 'diagonal' elements only.

For a partition $\lambda=\left\{\lambda_{1}, \lambda_{2}, \ldots, \lambda_{q}\right\}$ of $n$ we let

$$
f_{\lambda}(a, a, \ldots, a)=f\left(a^{\left|\lambda_{1}\right|}\right) f\left(a^{\left|\lambda_{2}\right|}\right) \ldots f\left(a^{\left|\lambda_{q}\right|}\right)
$$

and $\epsilon(\lambda)$ is the sign of a permutation whose cycle decomposition consists of cycles of lengths $\left\{\lambda_{1}, \lambda_{2}, \ldots, \lambda_{q}\right\}$. Hence, from (2.1) 


$$
\Phi_{n}(f)(a, a, \ldots, a)=\sum_{\lambda} \epsilon(\lambda) n(\lambda) f_{\lambda}(a, a, \ldots, a)
$$

where $n(\lambda)$ denotes the number of elements of the symmetric group $\Sigma_{n}$ in the conjugacy class determined by $\lambda$.

\section{(2.11) Lemma}

$$
\Phi_{n}(f)(a, a, \ldots, a)=(n-1) ! \sum_{k=1}^{n}(-1)^{k+1} f\left(a^{k}\right) \frac{\Phi_{n-k}(f)(a, a, \ldots, a)}{(n-k) !}
$$

Proof

This is obtained from Definition (2.1) by breaking the sum

$$
\Phi_{n}(f)(a, a, \ldots, a)=\sum_{\sigma \in \Sigma_{n}} \epsilon(\sigma) f_{\sigma}(a, a, \ldots, a)
$$

into parts corresponding to the length of the cycle in the permutation $\sigma$ that contains $n$ and there are $(n-1)(n-2) \ldots(n-k+1)$ such cycles of length $k$.

(2.12) Corollary The exponential generating function

$$
\sum_{n=0}^{\infty} \frac{\Phi_{n}(f)(a, a, \ldots, a)}{n !} t^{n}=\exp \left(\sum_{k=1}^{\infty}(-1)^{k+1} \frac{f\left(a^{k}\right)}{k} t^{k}\right)
$$

Proof This follows from the following well known combinatorial result.

(2.13) Lemma If $\Phi_{0}=1$ and

$$
\Phi_{n}=(n-1) ! \sum_{k=1}^{n} \frac{s_{k} \Phi_{n-k}}{(n-k) !} \text { for } n \geq 1
$$

then

$$
\sum_{n=0}^{\infty} \frac{\Phi_{n}}{n !} t^{n}=\exp \left(\sum_{k=1}^{\infty} \frac{s_{k}}{k} t^{k}\right)
$$

$\underline{\text { Proof }}$

Let $\Phi(t)=\sum_{n=0}^{\infty} \frac{\Phi_{n}}{n !} t^{n}$ and $s(t)=\sum_{k=0}^{\infty} s_{k} t^{k}$

then the hypothesis gives that

$$
t \Phi^{\prime}(t)=\Phi(t) s(t)
$$


SO

$$
\log (\Phi(t))=\int \frac{s(t)}{t}
$$

Checking the constant term gives the required conclusion.

Proof of (2.9)

We first note that by definition a map $f$ is a Frobenius $n$-homomorphism if and only if the exponential generating series is a polynomial of degree $n$ and $f(1)=n$. Using (2.12) we get that

$$
\sum_{k=0}^{\infty} \frac{\Phi_{k}(f+g)(a, a, \ldots, a)}{k !}=\sum_{r=0}^{m} \frac{\Phi_{r}(f)(a, a, \ldots, a)}{r !} \sum_{s=0}^{n} \frac{\Phi_{s}(g)(a, a, \ldots, a)}{s !}
$$

so $\Phi_{r}(f+g)(a, a, \ldots, a)=0$ for $r>m+n$ and clearly $(f+g)(1)=m+n$.

We will be interested in the behaviour of Frobenius $n$-homomorphisms on idempotent elements in $A$, and the following result is a generalisation of Corollary (2.5).

(2.14) Lemma If $B$ is a domain and $a \in A$ satisfies $a^{2}=a$ and $\Phi_{n+1}(f)(a, a, \ldots, a)=0$, then $f(a)=k$ for some integer $k$ such that $0 \leq k \leq n$.

$\underline{\text { Proof }}$

If $n=1$, then since $\Phi_{2}(f)(a, a)=0, f(a)^{2}=f\left(a^{2}\right)$ and so $f(a)(f(a)-1)=0$. If $\Phi_{n+1}(f)(a, a, \ldots, a)=0$, and $a^{2}=a$ then

$$
f(a) \Phi_{n}(f)(a, a, \ldots, a)-n \Phi_{n}(f)(a, a, \ldots, a)=0 .
$$

So, either, $f(a)=n$ or $\Phi_{n}(f)(a, a, \ldots, a)=0$ and the result follows by induction.

(2.15) Lemma If $a \in A$ then

$\Phi_{n}(f)(a, 1, \ldots, 1)=f(a)(f(1)-1)(f(1)-2) \ldots(f(1)-(n-1))$.

$\underline{\text { Proof }}$

This is straightforward to check for $n=1,2$ and then by induction.

\section{$\S 3$ Symmetric products}

In this section we relate our theory to the study of symmetric products.

The set of all Frobenius $n$-homomorphisms from $A$ to $\mathbf{C}$ will be denoted by $\Phi_{n}(A)$. 
Although we will prove a more general version of the following result, it is worth starting with the following simple proof.

(3.1) Theorem For a finite set $X$, the evaluation map

$$
\mathcal{E}: \operatorname{Sym}^{n}(X) \rightarrow \operatorname{Hom}(C(X), \mathbf{C})
$$

defined by $\left[x_{1}, x_{2}, \ldots, x_{n}\right] \rightarrow\left\{f \rightarrow \sum f\left(x_{r}\right)\right\}$ is an isomorphism onto the set of Frobenius n-homomorphisms.

$\underline{\text { Proof }}$

First note that evaluation at a point is a ring homomorphism $C(X) \rightarrow \mathbf{C}$ and so $\mathcal{E}\left[x_{1}, x_{2}, \ldots, x_{n}\right]$ is a Frobenius $n$-homomorphism by Corollary (2.10). Let $D=\left[x_{1}, x_{2}, \ldots, x_{n}\right] \in \operatorname{Sym}^{n}(X)$ be considered as a formal sum $D=$ $\sum m_{r} x_{r}$, where $m_{r} \in \mathbf{Z}_{+}$and $\sum m_{r}=n$. Let $e_{r} \in C(X)$ be the function that is 1 on $x_{r}$ and 0 elsewhere. Then $\mathcal{E}(D) e_{r}=m_{r}$.

Now if $f: C(X) \rightarrow \mathbf{C}$ is a Frobenius $n$-homomorphism and not an $(n-1)$ homomorphism, then, by (2.14), $f\left(e_{r}\right)=f_{r} \in \mathbf{Z}_{+}$and because 1 is the sum of the idempotents $e_{r}, \sum f_{r}=n$. Then $D=\sum f_{r} x_{r}$ is mapped onto $f$ by $\mathcal{E}$.

The map $\mathcal{E}$ is injective for a very general class of algebras of functions on a space $X$ (those where functions separate points) and so it is an isomorphism in the case of finite sets.

\section{(3.2) Theorem}

If $f: \mathbf{C}\left[u_{1}, u_{2}, \ldots, u_{m}\right] \rightarrow \mathbf{C}$ is a Frobenius $n$-homomorphism then there are points $\mathbf{x}_{1}, \mathbf{x}_{2}, \ldots \mathbf{x}_{n} \in \mathbf{C}^{m}$ such that $f(p)=p\left(\mathbf{x}_{1}\right)+p\left(\mathbf{x}_{2}\right)+\ldots+p\left(\mathbf{x}_{n}\right)$.

$\underline{\text { Proof }}$

By Theorem (2.8), the map

$$
\frac{\Phi_{n}(f)}{n !}: \mathcal{S}^{n}\left(\mathbf{C}\left[u_{1}, u_{2}, \ldots, u_{m}\right]\right) \rightarrow \mathbf{C}
$$

is a ring homomorphism. But $\mathcal{S}^{n}\left(\mathbf{C}\left[u_{1}, u_{2}, \ldots, u_{m}\right]\right)$ is the algebra of polynomial functions on $\operatorname{Sym}^{n}\left(\mathbf{C}^{m}\right)$, so $\frac{\Phi_{n}(f)}{n !}$ is given by evaluation at a multi-set $\left[\mathbf{x}_{1}, \mathbf{x}_{2}, \ldots, \mathbf{x}_{n}\right] \subset \mathbf{C}^{m}$. If $p \in \mathbf{C}\left[u_{1}, u_{2}, \ldots, u_{m}\right]$ then, on the one hand, by Theorem (2.8),

$\frac{\Phi_{n}(f)}{n !}((p, 1,1, \ldots, 1)+(1, p, 1, \ldots, 1)+\ldots+(1,1, \ldots, p))=p\left(x_{1}\right)+p\left(x_{2}\right)+\ldots+p\left(x_{n}\right)$

and, on the other hand, by the definition of $\Phi_{n}(f)$, the fact that $f(1)=n$ and Lemma (2.15) one gets that

$$
\frac{\Phi_{n}(f)}{n !}((p, 1,1, \ldots, 1)+(1, p, 1, \ldots, 1)+\ldots+(1,1, \ldots, p))=f(p) .
$$


This proves Theorem (3.2).

Reformulating this result and denoting the set of all Frobenius $n$-homomorphisms $f: \mathbf{C}\left[u_{1}, u_{2}, \ldots, u_{m}\right] \rightarrow \mathbf{C}$ by $\Phi_{n}\left(\mathbf{C}^{m}\right)$ gives

\section{(3.3) Corollary}

The evaluation map

$$
\mathcal{E}: \operatorname{Sym}^{n}\left(\mathbf{C}^{m}\right) \rightarrow \Phi_{n}\left(\mathbf{C}^{m}\right)
$$

is a homeomorphism.

\section{(3.4) Theorem}

Let $A$ be a finitely generated commutative algebra and let $f: A \rightarrow \mathbf{C}$ be a Frobenius n-homomorphism then there are ring homomorphisms $f_{i}: A \rightarrow \mathbf{C}$ for $1 \leq i \leq n$ such that $f=f_{1}+f_{2}+\ldots+f_{n}$.

$\underline{\text { Proof }}$

We deduce this from Theorem (3.2) (which is the special case when $A$ is a polynomial algebra). Let $q: \mathbf{C}\left[u_{1}, u_{2}, \ldots, u_{m}\right] \rightarrow A$ be a quotient map onto $A$ whose kernel we denote by $I$. Then $g=f q$ is a Frobenius $n$-homomorphism on $\mathbf{C}\left[u_{1}, u_{2}, \ldots, u_{m}\right]$ and so, by Theorem (3.2), there are ring homomorphisms $g_{i}: \mathbf{C}\left[u_{1}, u_{2}, \ldots, u_{m}\right] \rightarrow \mathbf{C}$ with $g=g_{1}+g_{2}+\ldots+g_{n}$. We show that each $g_{i}$ vanishes on $I$.

First we relabel the $g_{i}$ so that $g_{1}, g_{2}, \ldots, g_{k}$ are distinct and

$$
g=r_{1} g_{1}+r_{2} g_{2}+\ldots r_{k} g_{k} \text { for } r_{i} \in \mathbf{N} \text {. }
$$

If $\theta$ is a polynomial in $u_{1}, u_{2}, \ldots, u_{m}$, then $g_{i}(\theta)=\theta\left(g_{i}\left(u_{1}\right), g_{i}\left(u_{2}\right), \ldots, g_{i}\left(u_{m}\right)\right)$ since $g_{i}$ is a ring homomorphism, and if $\psi$ is another such polynomial $g_{i}(\psi \theta)=$ $g_{i}(\psi) g_{i}(\theta)$. If $\theta \in I$ then $g(\theta)=0$ and $g(\psi \theta)=0$ so

$$
\left(\begin{array}{cccc}
1 & 1 & \ldots & 1 \\
g_{1}(\psi) & g_{2}(\psi) & \ldots & g_{k}(\psi) \\
\vdots & \ddots & \ddots & \vdots \\
\vdots & \ddots & \ddots & \vdots \\
g_{1}(\psi)^{k-1} & g_{2}(\psi)^{k-1} & \ldots & g_{k}(\psi)^{k-1}
\end{array}\right)\left(\begin{array}{c}
r_{1} g_{1}(\theta) \\
r_{2} g_{2}(\theta) \\
\vdots \\
\vdots \\
r_{k} g_{k}(\theta)
\end{array}\right)=\left(\begin{array}{c}
0 \\
0 \\
\vdots \\
\vdots \\
0
\end{array}\right)
$$

\section{(3.5) Lemma}

Given distinct linear maps

$$
g_{1}, g_{2}, \ldots, g_{k}: \mathbf{C}\left[u_{1}, u_{2}, \ldots, u_{m}\right] \rightarrow \mathbf{C}
$$


there is a $\psi \in \mathbf{C}\left[u_{1}, u_{2}, \ldots, u_{m}\right]$ such that $g_{1}(\psi), g_{2}(\psi), \ldots, g_{k}(\psi) \in \mathbf{C}$ are distinct.

$\underline{\text { Proof }}$

By the assumption that the maps are distinct, $\operatorname{Ker}\left(g_{i}-g_{j}\right)$ has codimension one for every pair $i \neq j$. Any $\psi \notin \bigcup_{i \neq j} \operatorname{Ker}\left(g_{i}-g_{j}\right)$ will do.

For such a $\psi$, the matrix above is non-singular and so $r_{1} g_{1}(\theta)=r_{2} g_{2}(\theta)=$ $\ldots=r_{k} g_{k}(\theta)=0$. Hence each $g_{i}$ vanishes on $I$ and so defines a map $f_{i}$ on $A$ such that $f=r_{1} f_{1}+r_{2} f_{2}+\ldots r_{k} f_{k}$, that is $f$ is the sum of $n$ ring homomorphisms.

Remark Hence a set of distinct ring homomorphisms $\left\{g_{1}, \ldots, g_{k}\right\}$ gives a set of distinct vectors $\left\{v_{1}, \ldots, v_{k}\right\}$ and there is a polynomial $\Psi$, such that the numbers $\Psi\left(v_{i}\right)=g_{i}(\Psi)$ are distinct.

\section{(3.6) Corollary}

Let $A$ be a finitely generated commutative algebra and $V=\Phi_{1}(A)$. Then the evaluation map $\operatorname{Sym}^{n}(V) \rightarrow \Phi_{n}(A)$ is an isomorphism of varieties.

If $X$ is a compact Hausdorff space, let $\operatorname{Sym}^{n}(X)$ denote the symmetric product $X^{n} / \Sigma_{n}$ and $\mathbf{C}(X)$ the algebra of continuous functions on $X$ then the 'evaluation' map

$$
\mathcal{E}: \operatorname{Sym}^{n}(X) \rightarrow \Phi_{n}(\mathbf{C}(X), \mathbf{C})
$$

defined by

$$
\left[x_{1}, x_{2}, \ldots, x_{n}\right] \rightarrow\left(g \rightarrow \sum g\left(x_{k}\right)\right)
$$

is an embedding. It is clear that $\mathcal{E}$ is natural and so, if $X$ admits a group action, $\mathcal{E}$ is equivariant. Then, using the Gelfand transform that $\Phi_{1}(\mathbf{C}(X), \mathbf{C}) \cong$ $X$ one sees that this is a special case of the general question whether $\operatorname{Sym}^{n}(\Phi(A, B)) \rightarrow$ $\Phi_{n}(A, B)$ is an isomorphism when $B$ is any commutative domain.

We have already shown that when $X$ is a finite set the map $\mathcal{E}$ is onto.

(3.7) Theorem If $X$ is a compact Hausdorff space and the function space $\mathbf{C}(X)$ has the supremum norm, then the map

$$
\mathcal{E}: \operatorname{Sym}^{n}(X) \rightarrow \Phi_{n}^{c}(\mathbf{C}(X), \mathbf{C})
$$

is a homeomorphism when the space of continuous linear functionals on $\mathbf{C}(X)$ has the weak topology.

(3.8) Corollary Under these conditions every continuous Frobenius $n$ homomorphism is the sum of $n$ continuous ring homomorphisms. 
Proof of (3.7)

This is an easy adaptation of the proof of (3.4). If $f: C(X) \rightarrow \mathbf{C}$ is a continuous Frobenius $n$-homomorphism, then it is easily checked that

$$
\Phi_{n}(f) / n !: \mathcal{S}^{n}(C(X)) \rightarrow \mathbf{C}
$$

is a continuous ring homomorphism. But $\mathcal{S}^{n}(C(X))$ is isomorphic to the algebra $C\left(\operatorname{Sym}^{n}(X)\right)$ and the result follows as in (3.4).

Remark The case $n=1$ is the classical Gelfand transform map. However, it seems that the 'standard' proofs that the Gelfand map is an isomorphism do not adapt to prove this more general result. Indeed most of the proofs for the classical case $n=1$ do not 'find' the point of $X$ at which the ring homomorphism $\mathbf{C}(X) \rightarrow \mathbf{C}$ is evaluation. In another paper we will give such a constructive proof of (3.7).

\section{Acknowledgements}

We are very grateful to John McKay for pointing out the relevant papers by Frobenius and to Mark Haiman who explained to us that $\Phi_{n}$ is the expression for a multinomial symmetric function in terms of the power sums that we use, which lead to these proofs of the main theorems. We are also grateful to John Byatt-Smith for a helpful conversation and to Kostya Feldman for helpful comments on an earlier draft.

The research on which this is based was mainly carried out during visits by VMB to the University of Edinburgh and supported by the Engineering and Physical Sciences Research Council and the London Mathematical Society. Some of it was carried out at the Programme on Singularity Theory at the Isaac Newton Institute, Cambridge. 


\section{References}

[BR1] Buchstaber V. M. and E. G. Rees, Multi-valued groups, their representations and Hopf algebras, Transformation groups Vol 1 (1997), 325 -349, Birkhauser-Boston.

[BR2] Buchstaber V. M. and E. G. Rees, Multi-valued groups, $n$-Hopf algebras and $n$-ring homomorphisms, Chapter in Lie groups and Lie algebras (1998) 85 - 107, Kluwer Academic Publisher.

[BR3] Buchstaber V. M. and E. G. Rees, Frobenius $k$-characters and $n$ ring homomorphisms. (Russian) Uspekhi Mat. Nauk 52 (1997), no. 2(314),159-160; translation in Russian Math. Surveys 52 (1997), no. $2,398-39$

[For] Formanek E., The polynomial identities and invariants of $n \times n$ matrices, Amer. Math. Soc. (1991).

[Fro1] Frobenius G., Über Gruppencharaktere, Sitzungber. Preuss. Akad. Wiss. Berlin (1896), 985-1021.

[Fro2] Frobenius G., Über die Primfaktoren der gruppendeterminante. Sitzungber. Preuss. Akad. Wiss. Berlin (1896), 1343-1382.

[HJ] Hoehnke H.-J. and K. W. Johnson, The 1-, 2- and 3-characters determine a group, Bull. Amer. Math. Soc. 27 (1992) 243-245.

[Joh] Johnson K. W., On the group determinant, Math. Proc. Camb. Phil. Soc. 109 (1991) 299-311.

[W] Weyl, H., The Classical Groups, Princeton Univ. Press, (1946)

Dept. of Mathematics and Mechanics, Dept. of Mathematics and Statistics, Moscow State University, 119899 , James Clerk Maxwell Building, Moscow, King's Buildings,

Russia. Edinburgh EH9 3JZ, Scotland.

buchstab@mech.math.msu.su

elmer@maths.ed.ac.uk 